\title{
EFICIÊNCIA REPRODUTIVA DE FÊMEAS PRIMÍPARAS DA RAÇA NELORE
}

\author{
Alysson Oliveira Resende ${ }^{1}$, Carla Cristian Campos ${ }^{1}$, Mayara Oliveira ${ }^{1}$, Ricarda Maria \\ dos Santos ${ }^{1}$
}

1 UFU

Correspondência: Carla Cristian Campos: carlacristian_vet@yahoo.com.br

\begin{abstract}
RESUMO: Objetivou-se avaliar os efeitos da categoria animal (fêmeas primíparas de dois e de três anos comparadas com vacas multíparas) sobre a duração do intervalo entre o início da estação de monta (EM) e a concepção, o número de inseminações realizadas por fêmea e a taxa de gestação ao final da EM. Os animais foram submetidos a um protocolo de inseminação artificial em tempo fixo (IATF) com duração de 11 dias. O diagnóstico de gestação foi realizado entre 28 e 42 dias pós-IA e a confirmação da gestação entre 43 e 57 dias pós-IA, ambos por ultrassonografia. Os efeitos da duração do intervalo entre o início da EM e a concepção, bem como do número de inseminações de cada categoria foram avaliados por análise de variância, e a taxa de gestação ao final da EM por regressão logística, ambos no programa Minitab. A taxa de gestação geral foi de 70,59\%. Primíparas de dois anos tiveram $62,79 \%$ de taxa de gestação, enquanto que primíparas de três anos e as vacas multíparas tiveram $66,25 \%$ e $72,28 \%$, respectivamente. O intervalo do início da $E M$ até a concepção $(P=0,383)$ e a taxa de gestação $(P=0,182)$ não foram afetados pela categoria animal, porém foi detectado efeito desta variável sobre o número de inseminações por fêmea $(P=0,011)$. Primíparas de dois anos receberam, em média, 1,39 inseminações, enquanto que as de três anos receberam 1,86 e as multíparas 1,78 inseminações na EM. O menor número de inseminações nas primíparas precoces pode ser explicado pelo maior atraso no retorno à ciclicidade após o parto. Conclui-se que a categoria animal não afeta a duração do intervalo entre o início da estação de monta e a concepção, nem a taxa de gestação, porém vacas primíparas precoces de dois anos apresentam menor número de inseminações em relação às primíparas de três anos e as vacas multíparas.

Palavras-chave: bovinos; IATF; idade ao primeiro parto; puberdade
\end{abstract}

\section{REPRODUCTIVE EFFICIENCY OF EARLY NELORE PRIMIPAROUS FEMALES}

\begin{abstract}
This study aimed to evaluate the effects of animal category (early two years old primiparous, three years primiparous and multiparous cows) on interval duration between the onset of breeding season (BS) and conception, number of inseminations per female and pregnancy rate at the end of BS. Animals were submitted to a timed artificial insemination (TAl) protocol with 11 days of duration. The pregnancy diagnosis was done between 28 and 42 days post $\mathrm{Al}$ and the pregnancy confirmation between 43 and 57 days post $\mathrm{Al}$, both by ultrasonography. The effects of interval duration between the onset of BS and conception, as well as the number of inseminations of each category were evaluated by analysis of variance, and the pregnancy rate at the end of BS by logistic regression, both in Minitab program. The overall pregnancy rate was $70.59 \%$. Two years old primiparous had $62.79 \%$ of pregnancy rate, while three years primiparous and multiparous cows had $66.25 \%$ and $72.28 \%$, respectively. The interval between the onset of $B S$ and conception $(P=0.383)$ and pregnancy rate $(P=0.182)$ were not affected by animal category, however the effect of this variable was detected on number of inseminations per female $(P=0.011)$. Early primiparous received on average 1.39 inseminations, while three years received 1.86 and multiparous 1.78 inseminations in BS. The lowest number of inseminations in early primiparous can be explained by the delay on cyclicity return after calving. In conclusion animal category did not affect the interval duration between the onset of breeding season and conception neither pregnancy rate, however early two years old primiparous cows have fewer inseminations in relation of three years primiparous and multiparous cows.
\end{abstract}

Key Words: age at first calving; bovine; puberty; TAI 


\section{INTRODUÇÃO}

A idade à puberdade é uma característica determinante de produtividade na pecuária. A antecipação da idade ao primeiro parto (IPP) está diretamente ligada à eficiência e a lucratividade da cadeia produtiva da carne (DIAS et al. 2004), já que qualquer fator que atrase a ocorrência do primeiro estro e, consequentemente a concepção e o parto, acarreta perdas econômicas para o sistema.

De acordo com Ball e Peters (2006), em fêmeas bovinas a puberdade é o momento em que ocorre o primeiro estro acompanhado pela ovulação, ou seja, é quando a novilha adquire capacidade de se reproduzir, resultado de inúmeras alterações endócrinas, fisiológicas e morfológicas que a permitem conceber, manter a gestação e parir uma cria saudável. A idade à puberdade varia de acordo com fatores tais como peso corporal, estado nutricional, genética e características do manejo adotado.

Um fator que limita a eficiência produtiva dos rebanhos de corte no Brasil é o fato de que a maioria das novilhas da raça Nelore só produz sua primeira cria aos três ou quatro anos de idade (DAY et al., 2013). A redução da IPP de três para dois anos é capaz de aumentar em até $16 \%$ o retorno econômico da atividade. Assim, a vaca que pare aos dois anos produz um bezerro a mais, além de excluir um ano de recria, período no qual o animal representa apenas custos de produção sem geração de receita (DIAS et al., 2004; FERRAZ e ELER, 2008).

A idade a puberdade é uma importante característica produtiva em novilhas que são selecionadas para parir aos dois anos de idade, a fim de aumentar sua vida reprodutiva sem detrimentos à longevidade ou ao peso a desmama de suas crias, comparados àquelas que parem aos três anos (Tran et al., 1988). Porém, as novilhas que parem aos dois anos exibem um intervalo prolongado no pós-parto até o reinício dos ciclos estrais devido à elevada demanda energética para mantença, crescimento e lactação (MOSSMAN e HANLY, 1977; PATTERSON et al., 1992; SPITZER et al., 1995).

Vários hormônios são utilizados para induzir ciclicidade e antecipar a primeira ovulação pós-parto em primíparas a fim de melhorar o desempenho reprodutivo desta categoria (MENEGHETTI e VASCONCELOS, 2008). A inseminação artificial em tempo fixo (IATF) surge como uma alternativa para contornar os problemas relacionados às falhas na detecção do estro e ao prolongamento do anestro pós-parto, especialmente nas primíparas, já que esta categoria prioriza a energia disponível para o desenvolvimento corporal em detrimento à reprodução.

Este trabalho foi conduzido com o objetivo de avaliar os efeitos da categoria animal (primíparas precoces de dois anos de idade, primíparas de três anos e vacas multíparas) sobre a duração do intervalo entre o início da estação de monta (EM) e a concepção, o número de inseminações realizadas por fêmea e a taxa de gestação ao final da EM.

\section{MATERIAL E MÉTODOS}

O trabalho foi realizado em uma fazenda comercial localizada no município de Cachoeira Dourada, estado de Goiás, Brasil em uma EM de 94 dias entre os meses de dezembro de 2011 e março de 2012.

O rebanho da propriedade era composto por três categorias de matrizes puras de origem da raça Nelore, sendo utilizadas no experimento 43 primíparas de dois anos de idade, 80 
primíparas de três anos e 404 vacas multíparas, totalizando 527 matrizes avaliadas. As vacas eram mantidas em sistema de pastejo contínuo em pastagens formadas de Tifton 85 (Cynodon sp.) irrigadas, recebendo suplementação mineral e água $a d$ libitum.

O manejo reprodutivo da fazenda era realizado a cada 14 dias durante a $\mathrm{EM}$, no qual foram avaliadas a presença de corpo lúteo $(\mathrm{CL})$ e a involução uterina das vacas paridas acima dos 30 dias pós-parto, utilizando aparelho de ultrassonografia equipado com transdutor retal linear de 7,5Mhz (DP$3300 \mathrm{vet}^{\circledR}$, Mindray).

O escore de condição corporal (ECC) das vacas também foi avaliado dentro da escala de 1 a 5 (sendo 1 = muito magra e 5 = obesa) proposta por Houghton et al. (1990), estando aptas ao tratamento hormonal vacas cujo ECC fosse igual ou superior a 2,5.

As vacas foram submetidas ao seguinte protocolo de IATF: Dia zero (D0) - inserção do dispositivo intravaginal de liberação lenta de progesterona contendo $1,9 \mathrm{~g}$ deste hormônio (CIDR ${ }^{\circledR}$, Zoetis) e aplicação intramuscular de $2,0 \mathrm{~mL}$ de benzoato de estradiol (Estrogin ${ }^{\oplus}$, Farmavet); Dia 7 (D7) - aplicação de $2,5 \mathrm{~mL}$ de dinoprosttrometamina (Lutalyse ${ }^{\circledR}$, Zoetis); Dia 9 (D9) - retirada do dispositivo intravaginal e aplicação de $0,3 \mathrm{~mL}$ de cipionato de estradiol (E.C.P. ${ }^{\circledR}$, Zoetis) e de $1,5 \mathrm{~mL}$ de gonadotrofina coriônica equina (Folligon $\left.{ }^{\circledR}, M S D\right)$; Dia 11 - inseminação de todos os animais tratados. Todas as aplicações, inclusive a inseminação, foram realizadas no período da manhã.

O diagnóstico de gestação foi realizado entre os dias 28 e 42 pós-IA e a confirmação da gestação entre 43 e 57 dias pós-lA, ambos por ultrassonografia. As vacas vazias ao diagnóstico de gestação foram ressincronizadas utilizando o mesmo protocolo até se tornarem gestantes. Já as vacas que retornaram ao estro naturalmente no intervalo entre a inseminação e a data prevista para o diagnóstico de gestação foram reinseminadas convencionalmente, isto é, 12 horas após a detecção do estro. 0 manejo de observação do estro das fêmeas era realizado por funcionários treinados, duas vezes ao dia, no início da manhã e no final da tarde, durante cerca de 30 minutos.

Os efeitos da categoria animal (primíparas precoces de dois anos, primíparas de três anos e vacas multíparas) sobre a duração do intervalo entre o início da EM e a concepção, e sobre o número de inseminações realizadas por fêmea foram avaliados por análise de variância. Já o efeito da categoria animal sobre a taxa de gestação ao final da EM foi analisado por regressão logística, sendo que ambos os testes foram executados no programa Minitab. A significância estatística foi estabelecida como $\mathrm{P}<$ 0,05 .

\section{RESULTADOS E DISCUSSÃO}

A duração do intervalo entre o início da $E M$ e a concepção não foi influenciada $(P=0,383)$ pela categoria animal (Tabela 1), provavelmente porque 0 manejo nutricional $e$ reprodutivo adotado pela fazenda foi eficiente. As primíparas geralmente apresentam desempenho reprodutivo inferior em relação às multíparas devido às dificuldades na retomada da atividade reprodutiva no pós-parto. A elevada demanda energética desta categoria para atender seus requerimentos nutricionais de mantença, crescimento, lactação e reprodução (SPITZER et al., 1995) leva ao prolongamento do período de anestro pós-parto (MOSSMAN e HANLY, 1977; PATTERSON et al., 1992), porém este fato não foi verificado no presente estudo. 
Um estudo realizado por Oliveira et al. (2011) avaliou o número de dias pósparto na concepção após a IATF e também não constatou diferenças entre primíparas e multíparas da raça Nelore. Estes autores também demonstraram que a taxa de gestação variou entre primíparas e multíparas ao final da EM $(42,9 \%$ vs. $88,1 \%$, respectivamente), diferindo assim dos resultados encontrados no presente estudo (Tabela 1).

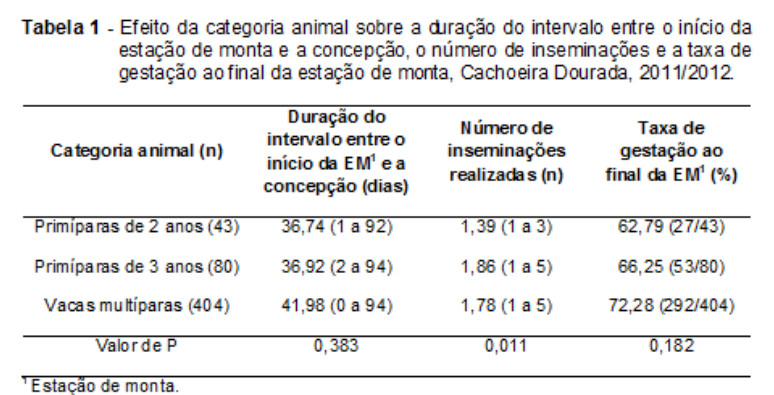

Foi detectado efeito $(P=0,011)$ da categoria animal sobre o número de inseminações realizadas por fêmea durante a EM. As primíparas precoces tiveram 0 número médio de 1,39 inseminações (Tabela 1). O número máximo de inseminações desta categoria foi igual a três, sendo todas elas em tempo fixo, já que nenhuma primípara de dois anos foi detectada em estro de retorno e, por isso, tiveram que ser ressincronizadas. Isto se deve, provavelmente, ao intenso balanço energético negativo sofrido no período pós-parto que influenciou negativamente a indução de ciclicidade pelo protocolo de IATF.

O número máximo de inseminações das primíparas de três anos e das multíparas foi igual a cinco (Tabela 1), considerando inseminações em tempo fixo e convencionais. Estes resultados podem ser explicados pelo fato de que algumas destas fêmeas foram detectadas em estro durante 0 manejo diário de observação e em seguida inseminadas, demonstrando a eficácia da IATF em induzir ciclicidade e em manter a regularidade dos ciclos estrais nas fêmeas que anteriormente estavam em anestro pós-parto (VASCONCELOS et al., 2006).

No presente estudo algumas vacas foram detectadas retornando ao estro após a IATF, indicando que estas fêmeas retornaram à atividade reprodutiva por consequência do tratamento hormonal. Já as fêmeas que não se tornaram gestantes e que também não foram detectadas em estro antes do diagnóstico de gestação, não conseguiram manter a regularidade dos ciclos estrais após o tratamento e, portanto permaneceram em anestro. Isto provavelmente explica o fato do número médio de inseminações ter sido menor nas primíparas precoces do que nas primíparas de três anos e nas vacas multíparas.

Das 527 matrizes utilizadas no experimento, 372 delas ficaram gestantes ao final da EM, resultando em uma taxa de gestação geral de $70,59 \%$. A taxa de gestação não foi afetada pela categoria animal $(P=0,182)$, sendo que $62,79 \%$ (27/43) das primíparas precoces de dois anos de idade ficaram gestantes ao final da EM, mostrando a viabilidade de se explorar a precocidade sexual de novilhas Nelore, pois a taxa de gestação desta categoria foi semelhante à encontrada para as demais categorias analisadas (Tabela 1).

Os resultados do presente estudo estão de acordo com os obtidos por Carneiro et al. (2012) que, ao submeter matrizes da raça Nelore à IATF por duas EM consecutivas, encontraram taxa de prenhez de $74,3 \%$ ao final da primeira EM e de 79,1\% após a segunda EM. Já Cunha et al. (2013) ao avaliar 350 vacas primíparas lactantes acíclicas da raça Nelore submetidas à monta natural e à IATF durante uma EM de 210 dias, obtiveram $46,57 \%$ de taxa de gestação ao final da EM, valor inferior ao encontrado no presente estudo. 
Explorar a precocidade sexual de novilhas zebuínas é uma forma de aumentar tanto a eficiência reprodutiva quanto a lucratividade da pecuária de corte. Segundo Teixeira et al. (2002) novilhas precoces que ficaram gestantes em torno dos 15 meses de idade resultaram em um acréscimo de $33,4 \%$ na receita bruta em relação às novilhas gestantes aos 27 meses, analisando a venda das crias destas matrizes. Ainda segundo estes autores, um dos problemas da intensificação da produção é a necessidade de proporcionar às fêmeas maiores ganhos de peso até a segunda cobertura, a fim de evitar uma queda na fertilidade destes animais na segunda EM.

A obtenção de resultados satisfatórios por meio da exploração da precocidade sexual de novilhas dependerá de um suporte nutricional e sanitário adequado para que estes animais não sejam acometidos pelo balanço energético negativo no pósparto, e da utilização de protocolos de sincronização da ovulação para indução da ciclicidade e realização da IATF.

\section{CONCLUSÃO}

Nas condições descritas neste experimento, a categoria animal não afetou a duração do intervalo entre o início da estação de monta e a concepção nem a taxa de gestação, porém vacas primíparas precoces de dois anos de idade apresentam menor número de inseminações em relação às primíparas de três anos e as vacas multíparas da raça Nelore.

\section{NOTAS INFORMATIVAS}

Número de protocolo aprovado pela Comissão de Ética na Utilização de Animais (CEUA) da Universidade Federal de Uberlândia (UFU) 025/13.

\section{REFERÊNCIAS}

BALL, P.J.H.; PETERS, A.R. Reprodução em bovinos. São Paulo, SP, 2006. 232p.

CARNEIRO, L.C.; CAMPOS, C.C.; SANTOS, R.M. Timed artificial insemination and early diagnosis of pregnancy to reduce breeding season in Nelore beef cows. Tropical Animal Health and Production, v.44, n.3, p.623-627, 2012.

CUNHA, R.R.; FERNANDES, C.A.C.; GARCIA, J.A.D. et al. Inseminação artificial em tempo fixo em primíparas Nelore lactantes acíclicas.

Arquivo Brasileiro de Medicina Veterinária e Zootecnia, v.65, n.4, p.1041-1048, 2013.

DAY, M.L.; CARVALHO, M.P.; CAMPOS, R.A.C. et al. Manejo nutricional para antecipar puberdade e prenhez em novilhas Nelore e cruzadas. In: CURSO NOVOS ENFOQUES NA PRODUÇÃO E REPRODUÇÃO DE BOVINOS, 17., 2013, Uberlândia. Anais...Uberlândia: CNEPRB, 2013, p.1-17. Disponível em: http://www.conapecjr.com.br/index.php/ Acesso em: 15/08/2013.

DIAS, L.T.; EL FARO, L.; ALBUQUERQUE, L.G. Estimativas de herdabilidade para a idade ao primeiro parto de novilhas da raça Nelore.

Revista Brasileira de Zootecnia, v.33, n.1, p.97-102, 2004.

FERRAZ, J.B.S.; ELER, J.P. Seleção para fertilidade em gado de corte. In: SIMPÓSIO INTERNACIONAL DE REPRODUÇÃO ANIMAL APLICADA, 3., 2008, Londrina. Anais... Londrina: SIRAA, 2008. p. 39-53. Disponível em: < http://www.geraembryo.com.br/artigos/3osimposio-internacional-de-reproducao-animalaplicada-arquivo-completo/ Acesso em: 02/04/2013.

HOUGHTON, P.L.; LEMENAGER, R.P.; MOSS, G.E. et al. Prediction of postpartum beef cow body composition using weight to height ratio and visual body condition score. Journal of Animal Science, v.68, p.1428-1437, 1990.

MENEGHETTI, M.; VASCONCELOS, J.L.M. Mês de parição, condição corporal e resposta ao protocolo de inseminação artificial em tempo fixo em vacas de corte primíparas. Arquivo

Brasileiro de Medicina Veterinária e Zootecnia, v.60, p.786-793, 2008.

MOSSMAN, D.H.; HANLY, G.J. A theory of beef production. New Zealand Veterinary Journal, v.25, p.96-100, 1977. 
OLIVEIRA, V.S.A.; BONATO, G.L.; SANTOS,

R.M. Eficiência reprodutiva de vacas primíparas da raça Nelore. Acta Scientiae Veterinariae, v.39, n.2, 963-966, 2011.

PATTERSON, D.J.; CORAH, L.R.; BRETHOUR, J.R. et al. Evaluation of reproductive traits in Bos taurus and Bos indicus crossbred heifers:

relationship of age at puberty to length of the postpartum interval to estrus. Journal of Animal Science, v.70, p.1994-1999, 1992.

SPITZER, J.C.; MORRISON, D.G.;

WETTERMAN, R.P.; FAULKNER, L.C.

Reproductive responses and calf birth and weaning weights as affected by body condition at parturition and postpartum weight gain in primiparous beef cows. Journal of Animal

Science, v. 73, n.5, p.1251-1257, 1995.

TEIXEIRA, R. A.; ALBUQUERQUE, L.G.;

FRIES, L.A. Comparações bioeconômicas entre três idades à primeira cobertura em novilhas Nelore. Ars Veterinaria, v.18, n.3, p.197-203, 2002.

TRAN, T.Q.; WARNICK, A.C., HAMMOND, M.E., KOGER, M. Reproduction in Brahman cows calving for the first time at two or three years of age. Theriogenology, v.29, p.751-756, 1988.

VASCONCELOS, J.L.M.; MENEGHETTI, M.; SANTOS R.M. Timed artificial insemination (TAI) in cows. Acta Scientiae Veterinariae, v.34

(Suppl 1), p.9-16, 2006. 